\title{
Nonlocality and Short-Range Wetting Phenomena
}

\author{
A. O. Parry, ${ }^{1}$ J. M. Romero-Enrique, ${ }^{1,2}$ and A. Lazarides ${ }^{1}$ \\ ${ }^{1}$ Department of Mathematics, Imperial College, 180 Queen's Gate, London SW7 2BZ, United Kingdom \\ ${ }^{2}$ Departamento de Física Atómica, Molecular y Nuclear, Area de Física Teórica, Universidad de Sevilla,
} Apartado de Correos 1065, 41080 Sevilla, Spain

(Received 6 May 2004; published 17 August 2004)

\begin{abstract}
We propose a nonlocal interfacial model for 3D short-range wetting at planar and nonplanar walls. The model is characterized by a binding-potential functional depending only on the bulk OrnsteinZernike correlation function, which arises from different classes of tubelike fluctuations that connect the interface and the substrate. The theory provides a physical explanation for the origin of the effective position-dependent stiffness and binding potential in approximate local theories and also obeys the necessary classical wedge covariance relationship between wetting and wedge filling. Renormalization group and computer simulation studies reveal the strong nonperturbative influence of nonlocality at critical wetting, throwing light on long-standing theoretical problems regarding the order of the phase transition.
\end{abstract}

DOI: 10.1103/PhysRevLett.93.086104

Density functional [1] and interfacial Hamiltonian models [2] are complementary approaches to the theory of confined fluids. Mean-field, nonlocal density functionals give an accurate description of structural properties but are unable to account correctly for long-wavelength interfacial fluctuations. To understand these it is usually necessary to employ mesoscopic interfacial Hamiltonians based on a collective coordinate $l(\mathbf{x})$, measuring the local interfacial thickness. These models are essentially local in character containing a surface energy term proportional to the stiffness $\Sigma$ of the unbinding interface and a binding-potential function $W(l)$. In more refined theories the stiffness also contains a position-dependent term [3], $\Sigma(l)$, which, it is has been argued, may drive the wetting transition first order [4]. Despite progress, over the last few years there are a number of outstanding problems particularly for wetting with short-ranged forces. In addition, recent studies of fluids in wedgelike geometries have uncovered hidden connections or wedge covariance relations between observables at planar wetting and wedge filling transitions [5], which have yet to be understood at a deeper level. In this Letter, we argue that analogous to developments in density functional methods the general theory of short-ranged threedimensional wetting should be formulated in terms of a nonlocal (NL) interfacial Hamiltonian. The model we propose directly allows for bulklike correlations arising from tubelike fluctuations [6] between the unbinding interface and the wall which contribute towards a binding-potential functional $W[l, \psi]$, where $\psi(\mathbf{x})$ is the shape function for the wall. Whilst the possible importance of such tubelike modes has been muted by several authors [7], to our knowledge this is the first quantitative treatment of them. Distinct contributions to $W[l, \psi]$ reflect the number of interacting tubes and have a simple diagrammatic interpretation. Three implications of the
PACS numbers: 68.08.Bc, 05.10.Cc, 05.70.Np, 68.35.Rh

NL model are discussed: (i) In the limit of small interfacial fluctuations, the NL model identically recovers the known form of the local Hamiltonian, allowing one to trace the specific position dependence of $W(l), \Sigma(l)$ to the Ornstein-Zernike (OZ) bulk correlation function. (ii) For filling in wedges with arbitrary tilt angles, the model obeys the classical wedge covariance relations observed in numerical studies of more microscopic theories. Such relations cannot be accounted for by local theories [8]. (iii) Renormalization group (RG) analysis shows the nonperturbative influence of NL interactions. Despite precise connection with the model and RG theory of Fisher and Jin (FJ) at perturbative level, the RG flow of the full NL model shows no stiffness instability and the wetting transition remains second order. Simulations confirm these findings.

Consider a Landau-Ginzburg-Wilson (LGW) Hamiltonian based on a continuum order-parameter (magnetization) $m(\mathbf{r})$ in a semi-infinite geometry with bounding surface described by a single-valued height function $\psi(\mathbf{x})$, where $\mathbf{x}=(x, y)$ is the parallel displacement vector. Denoting the surface magnetization by $m_{1}(\mathbf{x})$, we write

$$
H_{\mathrm{LGW}}=\int d \mathbf{r}\left[\frac{(\nabla m)^{2}}{2}+\phi(m)\right]+\int d s_{\psi} \phi_{1}\left(m_{1}\right),
$$

where $d s_{\psi}=\sqrt{1+(\nabla \psi)^{2}} d \mathbf{x}$ is the wall area element, while $\phi(m)$ and $\phi_{1}\left(m_{1}\right)$ are suitable bulk and surface potentials [9]. The bulk Hamiltonian is isotropic, so the interfacial tension and stiffness are the same. Following FJ we identify the interfacial model $H=H_{\mathrm{LGW}}\left[m_{\Xi}(\mathbf{r})\right]$, where $m_{\Xi}(\mathbf{r})$ is the profile which minimizes Eq. (1) subject to a given interfacial configuration. FJ determined $m_{\Xi}(\mathbf{r})$ perturbatively in terms of local planar constrained profiles [3]. Here we construct $m_{\Xi}$ nonperturbatively using Greens' functions or equivalently correlation func- 
tions defined within the constrained wetting layer. The latter reduces to the classical $\mathrm{OZ}$ form over relevant distances provided the wetting layer is much thicker than its bulk correlation length $\kappa^{-1}$. The NL Hamiltonian is

$$
H=\int d \mathbf{x}\left\{\Sigma \sqrt{1+(\nabla l)^{2}}+h(l-\psi)\right\}+W[l, \psi],
$$

where $h$ is proportional to the bulk field. There is no explicit position-dependent tension but rather a bindingpotential functional with three leading contributions:

$$
W[l, \psi]=-a \Omega_{1}^{1}[l, \psi]+b_{1} \Omega_{1}^{2}[l, \psi]+b_{2} \Omega_{2}^{1}[l, \psi],
$$

where $a, b_{1}$, and $b_{2}$ are best regarded as phenomenological parameters to be identified later. Each $\Omega_{\mu}^{\nu}$ represents integrated two-point interactions between $\mu$ and $\nu$ points on the wall and interface mediated by the (rescaled) bulk OZ correlation function $K(r)=\kappa e^{-\kappa r} / 2 \pi r$. These can be viewed as contributions to the free energy of a constrained thin film arising from tubelike fluctuations of the bulk phase which tunnel from the interface to the wall [6]. The first term involves only one tube:

$$
\Omega_{1}^{1}[l, \psi]=\int d s_{\psi}^{1} \int d s_{l}^{2} K\left(r_{12}\right)
$$

where $\quad d s_{l}^{\mu}=\sqrt{1+\left(\nabla l\left(\mathbf{x}_{\mu}\right)\right)^{2}} d \mathbf{x}_{\mu} \quad$ etc., $\quad$ and $\quad r_{12}=$ $\sqrt{\left|\mathbf{x}_{12}\right|^{2}+\left(\psi\left(\mathbf{x}_{1}\right)-l\left(\mathbf{x}_{2}\right)\right)^{2}}$ is the distance between two points on the interface and wall. The last two terms,

$$
\begin{aligned}
& \Omega_{1}^{2}[l, \psi]=\int d s_{\psi}^{1}\left\{\int d s_{l}^{2} K\left(r_{12}\right)\right\}^{2} \\
& \Omega_{2}^{1}[l, \psi]=\int d s_{l}^{2}\left\{\int d s_{\psi}^{1} K\left(r_{12}\right)\right\}^{2},
\end{aligned}
$$

involve two tubes and may be viewed as a self-interaction between points on the same interface or wall induced by the presence of a second surface. Each contribution can be represented diagrammatically as shown in Fig. 1. The upper and lower lines represent typical nonplanar configurations of the interface and wall. The undulated line joining them represents the interaction function $K\left(r_{12}\right)$, while the solid dots imply integration over the area of each surface. For general wall and interfacial configurations, all contributions to $W[l, \psi]$ are NL. Simplifications

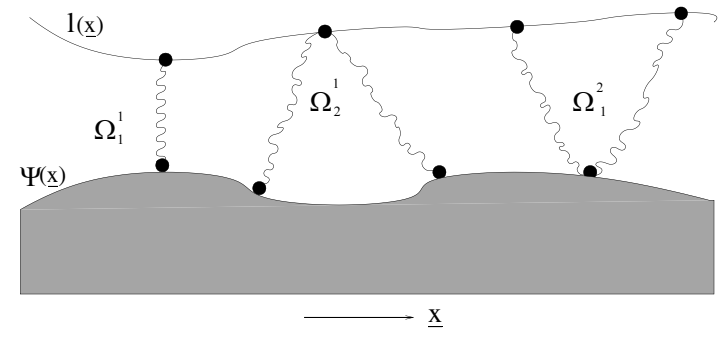

FIG. 1. Schematic illustration of the diagrams which represent the leading order contributions to $W[l, \psi]$. arise when one or both are planar. If both the wall and interface are flat, $\psi(\mathbf{x})=0, l(\mathbf{x})=l$ the Hamiltonian per unit area $W(l)=W[l, 0] / A$ reduces to

$$
W(l)=-a e^{-\kappa l}+\left(b_{1}+b_{2}\right) e^{-2 \kappa l},
$$

which recovers the standard form of the binding-potential appearing in local models. For the more general case of a nonplanar interface near a planar wall, two contributions to the binding-potential functional are local since

$$
\Omega_{\mu}^{1}[l, 0]=\int d s_{l}^{1} e^{-\mu \kappa l\left(\mathbf{x}_{1}\right)}, \quad \mu=1,2 .
$$

However, $\Omega_{1}^{2}$ remains NL and can be rewritten as a twobody repulsive interaction

$$
\Omega_{1}^{2}[l, 0]=\iint d s_{l}^{1} d s_{l}^{2} S\left(\left|\mathbf{x}_{12}\right| ; \bar{l}\right),
$$

where $\bar{l}=\left[l\left(\mathbf{x}_{1}\right)+l\left(\mathbf{x}_{2}\right)\right] / 2$, and $S(x ; \bar{l})$ is

$$
S(x ; \bar{l})=\frac{\kappa^{2}}{2 \pi} \int_{2 \kappa \bar{l}}^{\infty} d t \frac{e^{-\sqrt{t^{2}+\kappa^{2} x^{2}}}}{\sqrt{t^{2}+\kappa^{2} x^{2}}} \approx \frac{\kappa}{4 \pi \bar{l}} e^{-2 \kappa \bar{l}-\kappa x^{2} / 4 \bar{l}},
$$

valid for $\kappa \bar{l} \gg 1$. In the small gradient limit, $|\nabla l| \ll 1$, the NL term can be expanded and the model reduces to

$$
H[l, 0] \approx \int d \mathbf{x}\left\{\frac{\Sigma(l)}{2}(\nabla l)^{2}+W(l)\right\},
$$

with stiffness coefficient

$$
\Sigma(l)=\Sigma-a e^{-\kappa l}-2 b_{1} \kappa l e^{-2 \kappa l}+\cdots
$$

precisely recovering the FJ model and uniquely identifying $a, b_{1}$, and $b_{2}$. In particular, $a$ measures the deviation from the mean-field (MF) critical wetting temperature, $b_{2} \propto a^{2}$, and the sign of $b_{1}$ determines the order of the MF transition. Thus the origin of the $\kappa l e^{-2 \kappa l}$ contribution, crucial in the FJ analysis, can be traced directly to a perturbative treatment of the NL contribution $\Omega_{1}^{2}$.

Now consider fluid adsorption in a wedge geometry $(\psi=\tan \alpha|x|)$. The NL model satisfies the necessary requirement of classical wedge covariance known from numerical studies of the microscopic model (1) [8]. Classical wedge covariance refers to the relationship between observables at MF critical wetting and MF wedge filling transitions. Let $l_{\pi}(\theta)$ denote the MF thickness of the critical wetting layer written as a function of the contact angle. Let $l_{w}(\theta, \alpha)$ denote the thickness of the filling layer above the wedge midpoint. Numerical minimization of the LGW Hamiltonian (1) shows that $l_{w}(\theta, \alpha)=l_{\pi}(\theta-\alpha)$ as $\theta \rightarrow \alpha$, for both shallow and acute wedges. This relation cannot be explained using an approximate local Hamiltonian in which the interface interacts with the (closest) wall via a binding potential dependent on the normal distance [10]. Such models predict the incorrect behavior $l_{w}(\theta, \alpha)=\sec \alpha l_{\pi}(\theta-$ $\alpha$ ). In contrast, the NL model obeys the correct wedge 
covariant relation. The reason for this can be traced to the structure of the NL binding potential. Since filling precedes wetting $(a \neq 0)$, the dominant term is $\Omega_{1}^{1}$. Now for a flat interfacial configuration $l(\mathbf{x})=l_{0}$ near a nonplanar wall both $\Omega_{1}^{1}$ and $\Omega_{1}^{2}$ are local with, for example,

$$
\left.\Omega[l, \psi]\right|_{l=l_{0}}=\int d \mathbf{x} \sqrt{1+(\nabla \psi)^{2}} e^{-\kappa\left[l_{0}-\psi(\mathbf{x})\right]}
$$

showing that the effective local interaction occurs via the vertical distance to the surface. Near the filling phase boundary the interface is essentially flat in the filled section of the wedge and the $\Omega_{1}^{1}$ contribution must be of the above form. This is sufficient to ensure covariance. We also remark that for wetting at more general nonplanar walls the NL model reproduces the precise form of the stiffness matrix appearing in approximate two-field models [11] valid for $|\nabla l| \ll 1$ and $|\nabla \psi| \ll 1$. This means that in application to complete wetting the NL theory satisfies exact sum rules [12].

Finally, we turn to the controversy surrounding fluctuation effects at planar critical wetting. The standard capillary wave $(\mathrm{CW})$ model, obtained by setting $\Sigma(l) \equiv$ $\Sigma$ in Eq. (11), famously predicts nonuniversal criticality dependent on the wetting parameter $\omega=k_{B} T \kappa^{2} / 4 \pi \Sigma$ [13]. However, this strongly disagrees with Ising model simulation studies [14], which show only minor deviations from MF-like critical wetting behavior (for the experiments, see Ref. [15]). The more refined FJ model provides a possible explanation of this discrepancy, since the $\Sigma(l)$ term drives the transition first order for physical values of $\omega$ [4]. Here we show that the stiffness instability is not a robust mechanism, since the wetting transition described by the NL model remains continuous. A linear RG theory can be constructed provided we first expand $\sqrt{1+(\nabla l)^{2}}$ to square gradient order. The local terms $\Omega_{\mu}^{1}$ generate effective binding-potential and positiondependent stiffness contributions, which renormalize as in Refs. $[4,13]$. We focus on the renormalization of the NL potential $S(x ; \bar{l})$, which controls the order of the phase transition since it is responsible for the $-l e^{-2 \kappa l}$ term in the perturbative $|\nabla l| \ll 1$ limit. After renormalizing up to a scale $b=e^{t}$, the NL term $\Omega_{1}^{2}$ retains its two-body form but with a modified potential $S_{t}(x ; \bar{l})$ satisfying the flow equation:

$$
\frac{\partial S_{t}}{\partial t}=4 S_{t}+x \frac{\partial S_{t}}{\partial x}+\omega \kappa^{-2}\left(\frac{1+J_{0}(\Lambda x)}{2}\right) \frac{\partial^{2} S_{t}}{\partial \bar{l}^{2}},
$$

where $J_{0}(x)$ is a Bessel function of first kind and $\Lambda$ is the momentum cutoff. This equation has the formal solution:

$$
S_{t}(x ; \bar{l})=e^{4 t} \int_{-\infty}^{\infty} d l \frac{\kappa S_{0}\left(x e^{t}, l\right) \exp \left[-(\kappa l-\kappa \bar{l})^{2} / 4 \omega \Phi\left(\Lambda x e^{t}, \Lambda x\right)\right]}{\sqrt{4 \pi \omega \Phi\left(\Lambda x e^{t}, \Lambda x\right)}},
$$

where $\quad \Phi(a, b)=\int_{b}^{a} d t\left[1+J_{0}(t)\right] / 2 t$. We choose $S_{0}(x, l)=\Theta(l) S(x, l)$, with $\Theta(l)$ the Heaviside step function and $S(x, l)$ given by Eq. (10). As $t \rightarrow \infty, S_{t}(x, l)$ becomes increasingly localized around $x=0$. Using a matching technique, we renormalize to a scale $e^{t^{*}}$ at which the curvature of the effective binding-potential $W_{t}(l)$ at its global minimum is of order of $\Sigma \kappa^{2}$. Our $W_{t}(l)$ has a local contribution due to the $\Omega_{1}^{1}$ and $\Omega_{2}^{1}$ processes, and a NL contribution, which is obtained from the expansion of $\Omega_{1}^{2}$ in powers of $\nabla l$ :

$$
W_{t}^{\mathrm{NL}}(l)=2 \pi \int_{0}^{\infty} d x x S_{t}(x, l) .
$$

Numerical integration of the RG flow equation show that the wetting transition is always second order and quantitatively similar to the nonuniversality exhibited by the $\mathrm{CW}$ model. This fact can be rationalized by noting that $\Phi\left(\Lambda x e^{t}, \Lambda x\right) \sim t$ as $t \rightarrow \infty$ and $\kappa x \lessgtr e^{-t}$, which is the range where $S_{0}\left(x e^{t}, l\right)$ is non-negligible. Consequently, in our NL model there is no stiffness instability. The difference with the RG predictions of the FJ model arises specifically from nonlocality. Mathematically, the FJ flow equations can be recovered from Eq. (14) if we approximate the Bessel function term by its quadratic expansion in $x$. However, this is not valid at large distances and invalidates the stiffness instability.
In order to check the RG predictions, we have performed Monte Carlo simulations of the CW, FJ, and NL Hamiltonians [with the approximation $\sqrt{1+(\nabla l)^{2}} \approx 1+$ $\left.(\nabla l)^{2} / 2\right]$. Following Ref. [16], we discretize by introducing a $L \times L$ lattice of spacing $\sigma$ with periodic boundary conditions in the directions parallel to the surface but treating the interfacial position height as continuous variables. We chose $\sigma=3.1623 \kappa^{-1}$ so that $\Lambda \kappa^{-1} \sim \pi / \kappa \sigma \lesssim$ 1 , and also set $\omega=0.8$ and $b_{1}=2.5 \kappa^{2} k_{B} T$, which are reasonable Ising-like parameters. We anticipate that the critical wetting phase boundary remains $\operatorname{MF}(a=0)$ for the CW and NL theories [13], while the FJ exhibits a firstorder transition at higher temperatures [4]. Figure 2 describes the behavior of the mean wetting layer thickness $\langle l\rangle$ and the surface magnetizationlike operator $\Delta m_{1}=$ $\left\langle e^{-\kappa l}\right\rangle$ along the MF critical wetting isotherm $a=0, h \rightarrow$ 0 . The FJ model clearly describes partial wetting in this limit, consistent with a fluctuation-induced first-order transition. On the other hand, the CW and NL models are qualitatively similar, showing continuous wetting. The divergence of the film thickness is well described by the RG result $\kappa\langle l\rangle \sim-\sqrt{2 \omega} \ln h$, even for moderately thick wetting layers. However, the surface magnetization shows a much larger preasymptotic critical regime. The asymptotic nonuniversal behavior $\Delta m_{1} \sim h^{1-1 / 2 \nu_{\|}}$, with $\nu_{\|}=(\sqrt{2}-\sqrt{\omega})^{-2}$ is not observed until the wetting layer

086104-3 


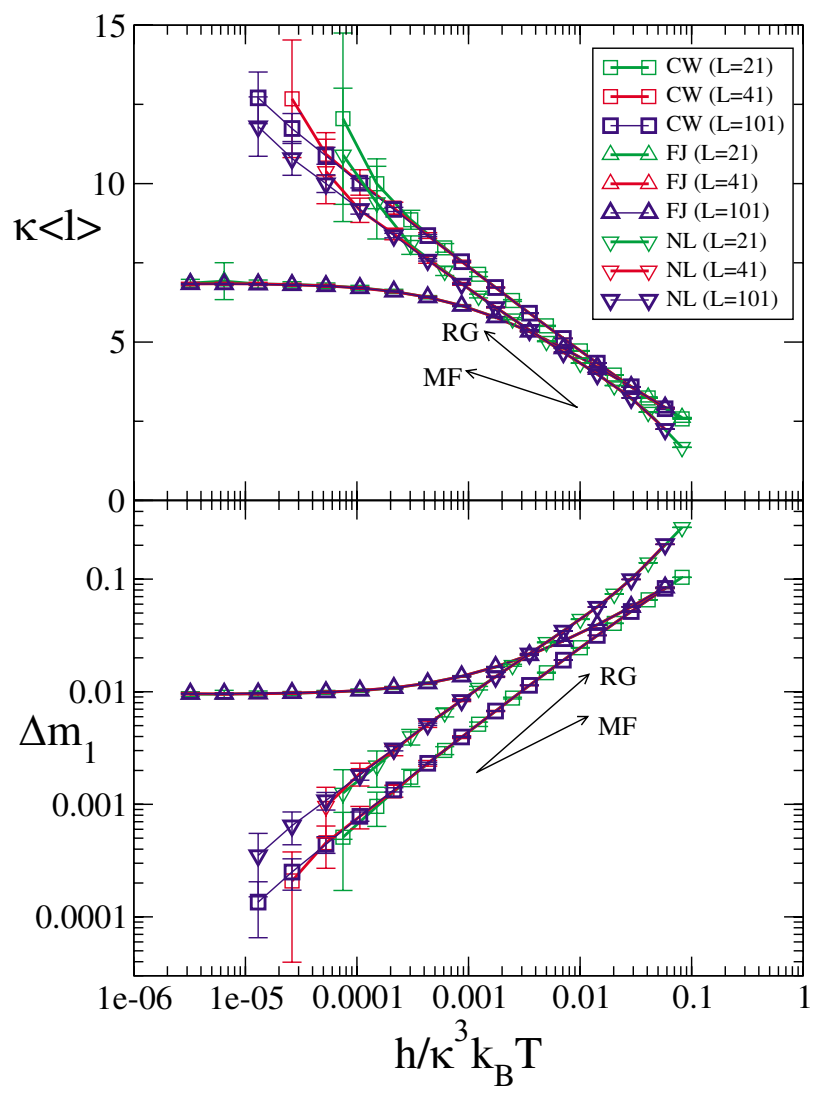

FIG. 2 (color online). Plot of the mean wetting layer $\langle l\rangle$ and surface magnetization operator $\Delta m_{1}$ versus $h$ obtained by computer simulations of the $\mathrm{CW}, \mathrm{FJ}$, and our NL model for $\omega=0.8, a=b_{2}=0$, and $b_{1} / \kappa^{2} k_{B} T=2.5$.

$\kappa\langle l\rangle \sim 10$ for very large lattice sizes $\kappa L \sim 300$. This is strongly suggesting that current Ising model simulations will not be able to observe significant deviation from MF behavior provided they focus on surface quantities.

We conclude by mentioning extensions and limitations of our theory. It is straightforward to generalize the model to describe wetting at heterogeneous substrates with, for example, hydrophilic and hydrophobic domains [17]. For such geometries, all the contributions to $W[l, \psi]$ will be NL and may influence mesoscopic wetting behavior. The same is true for other types of homogeneous sculpted substrates as, for example, wedges of parabolic cross section [18]. However, even for the simple case of wetting at homogeneous planar walls, systematic improvements of the theory can be envisaged. The binding-potential functional (3) is valid only for wetting layers many times larger than $\kappa^{-1}$. If the wetting layer is only a few bulk correlation lengths thick, the interaction function $K(r)$ should be replaced by the correlation function defined within the constrained profile. This would modify the form of the binding-potential functional at short distances and may influence how the asymptotic critical regime is approached.
A. O. P. gratefully acknowledges Professor D. B. Abraham for discussions regarding the origin of the binding potential. We also thank Professor M. E. Fisher and Dr. J. Gibbons for helpful comments. J. M. R.-E. acknowledges partial financial support from Secretaría de Estado de Educación y Universidades (Spain), cofinanced by the European Social Fund, and from the European Commission under Contract No. MEIF-CT-2003-501042. A. L. acknowledges partial financial support from the A. G. Leventis Foundation.

[1] See, for example, R. Evans, in Fundamentals of Inhomogeneous Fluids, edited by D. Henderson (Dekker, New York, 1992).

[2] G. Forgacs, R. Lipowsky, and Th. M. Nieuwenhuizen, in Phase Transition and Critical Phenomena, edited by C. Domb and J. L. Lebowitz (Academic Press, New York, 1991), Vol. 14.

[3] M. E. Fisher and A. J. Jin, Phys. Rev. B 44, 1430 (1991); A. J. Jin and M. E. Fisher, Phys. Rev. B 47, 7365 (1993).

[4] M. E. Fisher and A. J. Jin, Phys. Rev. Lett. 69, 792 (1992); A. J. Jin and M.E. Fisher, Phys. Rev. B 48, 2642 (1993); C. J. Boulter, Phys. Rev. Lett. 79, 1897 (1997).

[5] A. O. Parry, M. J. Greenall, and A. J. Wood, J. Phys. Condens. Matter 14, 1169 (2002).

[6] D. B. Abraham, J.T. Chayes, and L. Chayes, Commun. Math. Phys. 96, 439 (1984).

[7] D. B. Abraham (private communication); See also M. E. Fisher, in Statistical Mechanics of Membranes and Surface, edited by D. R. Nelson, T. Piran, and S. Weinberg (World Scientific, Singapore, 1989).

[8] M. J. Greenall, A. O. Parry, and J. M. Romero-Enrique, J. Phys. Condens. Matter 16, 2515 (2004).

[9] H. Nakanishi and M. E. Fisher, Phys. Rev. Lett. 49, 1565 (1982).

[10] K. Rejmer, S. Dietrich, and M. Napiorkowski, Phys. Rev. E 60, 4027 (1999).

[11] C. J. Boulter and A. O. Parry, Phys. Rev. Lett. 74, 3403 (1995).

[12] See J. R. Henderson, in Fundamentals of Inhomogeneous Fluids, edited by D. Henderson (Dekker, New York, 1992).

[13] E. Brézin, B. I. Halperin, and S. Leibler, Phys. Rev. Lett. 50, 1387 (1983); D. S. Fisher and D. A. Huse, Phys. Rev. B 32, 247 (1985).

[14] K. Binder, D. P. Landau, and D. M. Kroll, Phys. Rev. Lett. 56, 2272 (1986).

[15] D. Ross, D. Bonn, and J. Meunier, Nature (London) 400, 737 (1999).

[16] G. Gompper and D. M. Kroll, Phys. Rev. B 37, 3821 (1988).

[17] H. Gau et al., Science 283, 46 (1999).

[18] C. Rascón and A. O. Parry, Nature (London) 407, 986 (2000). 